\title{
Borges y la matemática
}

\author{
Borges and mathematics
}

\author{
Borges e a matemática
}

Hernando Gutiérrez Hoyos

Especialista en Matemáticas Avanzadas

Universidad Surcolombiana

herguho@usco.edu.co

\section{Resumen}

La creación Matemática y la creación Literaria tienen más afinidades y analogías que otras actividades que comprometan al cerebro humano. Muchos de los objetos y elementos sobre los cuales trabajan algunas teorías Matemáticas son verdaderas ficciones al igual que los objetos y los seres que protagonizan un cuento o una novela. Así, por ejemplo, los Alephs introducidos por Cantor para cuantificar los Conjuntos Infinitos (estableciéndose como cardinales Transfinitos) son retomados por el ilustre literato Jorge Luis Borges para muchas de sus construcciones narrativas en las que materializa y objetiva algunos infinitos como totalidades (en acto).

El rigor que acompaña el discurrir en la construcción de las teorías Matemáticas para garantizar la coherencia y evitar las contradicciones estructurales, es similar al rigor que el literato le imprime a su obra para hacerla consistente y darle credibilidad.

Palabras claves: Borges, Cantor, infinito potencial, infinito actual, Aleph.

\begin{abstract}
Mathematical creation and literary creation have more affinities and analogies than other activities that engage the human brain. Many of the objects and elements on which they have some mathematical theories are true fiction, as are the objects and beings that star in a story or novel. Thus, for example, the Alephs introduced by Cantor to quantify the Infinite Sets (establishing themselves as Cardinal Transfinite) are taken up by the illustrious writer Jorge Luis Borges for many of his narrative constructions in which he materializes and objectifies some infinities as totalities (in act).
\end{abstract}

The rigor that goes along with the construction of Mathematical theories to protect coherence and avoid structural contradictions is similar to the rigor that the writer places on his work to make it consistent and give it credibility. 
Keywords: Borges, Cantor, potential infinity, actual infinity, Aleph.

\section{Resumo}

A criação matemática e a criação literária têm mais proximidades e analogias que outras atividades do cérebro humano. Muitos dos objetos e elementos sobre os quais trabalham algumas teorias da matemática são verdadeiras ficções semelhantes aos objetos e personagens que protagonizam um conto ou um romance. Deste modo, os Aleph introduzidos pelo cantante para quantificar os Conjuntos Infinitos (estabelecidos como Cardinais Transfinitos) são retomados pelo ilustre literato Jorge Luís Borges para muitas das suas obras narrativas, nas quais materializa e objetiva alguns infinitos como totalidades (em ato).

O rigor que acompanha o decorrer na construção das teorias da matemática para garantir a coerência e evitar as contradições estruturais, é semelhante ao rigor que o literato coloca na sua obra para fazê-la consistente e acreditável.

Palavras-chave: Jorge Luis Borges, Cantor, infinito potencial, infinito atual, Aleph.

El propósito de esta reflexión es presentar evidencias sobre la existencia de analogías y afinidades entre la creación Literaria y la creación Matemática partiendo del manejo que en estos campos del conocimiento, y por iniciativa de reconocidos y acreditados cultores de las mismas, se le ha dado a algunas categorías genéricas que han sido objeto de estudio en las ciencias y la filosofía, además de inspiración artística e incluso material de especulaciones de todo tipo, incluyendo las místicas y esotéricas que son las más concurridas y favorecidas popularmente. Para tal fin he seleccionado algunos temas tan problemáticos y antiguos como el ser humano, que en Filosofía y Literatura han sido tratados con la discrecionalidad y la licencia propias de dichos campos del saber, mientras que en Matemática se les encierra en los esquemas del rigor y el formalismo inherentes a la disciplina.

El primer tema por abordar será el del Infinito. Seguramente un tema tan antiguo como el Homo Sapiens y generador de controversias en cualquier campo donde pretenda ser discutido. Para asumir este tema desde los frentes de trabajo amablemente confrontados en esta exposición, sin otra intención que la de buscar sus relaciones, la Literatura y las Matemáticas, he convocado a Jorge Luis Borges (Buenos Aires, Argentina, 1899; Ginebra, Suiza, 1986) y
George Cantor (San Petersburgo 1845; Alemania 1918) respectivamente. En representación del enfoque Literario se cita a Borges no sólo por ser uno de los mayores exponentes de la creación en este campo, sino por su cercanía con la Matemática. Borges, es necesario aclararlo, no es Matemático; ni siquiera podemos hablar (como lo creen algunos biógrafos y admiradores de Borges) de algo que pueda llamarse el Pensamiento Matemático de Borges. Lo que sí es incuestionable, es la profunda admiración y el respeto que este profesaba por la matemática. Se acercó a Ella para recrear, desde el rigor y la precisión, sus ideas sin renunciar a la ficción, la fantasía y la inventiva que constituyen la esencia de la creación literaria. Para él, en cierto sentido, la Matemática fue el aval de muchos de sus cuentos, relatos y ficciones.

En el enfoque Matemático estará Cantor, uno de los matemáticos más admirados, respetados y estudiados por Borges; tal vez por haber sido una mente lúcida, distanciada de los lugares comunes, contradictoria, obsesionada de las normas y convencionalismos que desde la Edad Media reducían la creación matemática al entorno de las concepciones escolásticas, y la primera en trascender las fronteras de lo finito para domesticar una de las bestias mitológicas de la Filosofía, la Lógica y las Matemáticas: el infinito. 
Esta búsqueda de evidencias que justifiquen analogías entre dos campos del conocimiento, aparentemente contradictorios, no sólo en cuanto a la naturaleza de los objetos de estudio sino a la validez de sus resultados y a la verdad de los mismos, encuentra en la sana confrontación de los haceres y saberes un elemento que no sólo supera la contradicción sino que constituye el fundamento mismo de la creación, tanto literaria como matemática, aproximando y hasta emparentando estos dos campos del conocimiento teniendo como base la libertad.

Así lo había proclamado Cantor para la Matemática: "La esencia de la Matemática es La Libertad". Los grandes creadores, que a través de la historia de la Matemática lo han sido y como tales los ha reconocido la comunidad académica, trabajaron en su intimidad, al igual que los "hacedores" de historias, cuentos, narraciones y ficciones, con absoluta libertad. En muchos de sus extraordinarios descubrimientos llegaron a resultados finales, a través de ingeniosos recursos y curiosos supuestos, que horrorizaron tanto a los cancerberos de la Escolástica como a los funcionarios grises de las dependencias encargadas de la inquisición y la censura del material educativo. Estimularon y exaltaron la rebeldía de las mentes juveniles atraídas por la belleza, la genialidad y la potencialidad de las nuevas formas argumentativas. Tal vez el más grande de todos fue Arquímedes, precursor de Cantor en la exploración del infinito mediante el recurso de las "cantidades" Infinitesimales que tanta atracción ejercieron en el prelado Nicolás de Cusa (1401-1464), referenciado por Borges en muchos de sus escritos, insigne pionero del moderno análisis matemático como explorador y constructor (en compañía de otras mentes tan lúcidas como la suya) de caminos que, siglos después, convergieron exitosamente en la construcción del concepto de límite como soporte del análisis matemático.

Desde los albores de aquellas civilizaciones donde las condiciones políticas, sociales, culturales y económicas permitían y estimulaban la libertad de pensamiento y el ejercicio intelectual, temas como el infinito, tanto en lo espacial como lo temporal, el eterno retorno, las regresiones infinitas, la circularidad como encuentro a perpetuidad del comienzo y el fin, la reproducción múltiple de imágenes en elementos materiales de superficies brillantes (los espejos, por ejemplo), las permutaciones, las combinaciones, los laberintos y las bifurcaciones, han sido objeto de interpretaciones que varían desde las especulaciones premonitorias y cabalísticas, que intuyen encriptado en estas categorías, por disposiciones divinas, el destino de los seres y las cosas que pueblan el universo, hasta las rigurosas construcciones intelectuales propuestas desde los campos de las ciencias y/o de la filosofía al igual que de encendidos debates filosóficos y científicos.

Precisamente, fueron las inquietudes intelectuales de Borges con respecto a estos temas, que lo obsesionan y se vuelven recurrentes en sus escritos, las que lo acercaron a la Matemática pues, como hombre culto que era, sabía que desde esta ciencia se había conceptualizado rigurosamente sobre dicha temática. Así, por ejemplo, al comienzo de su reflexión sobre los procesos infinitamente recurrentes que generaron las paradojas de Zenón, recogida en su relato: "Los avatares de la tortuga", manifiesta que:

Hay un concepto que es el corruptor y el desatinador de los otros. No hablo del mal cuyo limitado imperio es la ética; hablo del INFINITO. Yo anhele compilar alguna vez su móvil historia... Cinco, siete años de aprendizaje Metafísico, Teológico, Matemático, me capacitaron (tal vez) para planear decorosamente ese libro. Inútil agregar que la vida me prohíbe esa esperanza, y aún ese adverbio. p.254, 1974

Así pues, llega Borges a la Matemática, y lo hace de la mano de dos grandes (tal vez de los más grandes): Cantor, reconocido como el creador de la Teoría de Conjuntos, y Bertrand Russell, uno de los mejores y más profundos 
lógico-matemáticos del siglo XX (además, premio Nobel de literatura en 1950). Del primero estudió su teoría sobre la cardinalidad transfinita a través de la cual vio "domesticado" al monstruo que lo acechaba permanentemente y que a cambio de asustarlo le alimentaba y estimulaba sus fantasías y ficciones: El Infinito. Lo identificó convertido en un Aleph. Al segundo, lo paseó por el mundo de lo paradójico y, con su extraordinaria agilidad mental, le mostró cómo las paradojas emergen de las contradicciones internas alojadas en los principios o en las definiciones sobre los que se construye una teoría y le enseñó a presentar alternativas para blindarla ante un posible ataque con formulaciones paradójicas.

En la Grecia antigua, cuando los filósofos se ocuparon del infinito, se acogió inicialmente la caracterización del infinito como lo sin fin, lo insondable, lo inabarcable. Excelente oportunidad para que, con la consolidación de las religiones monoteístas, lo infinito se identificase con su Dios atribuyéndole a éste la infinitud como una de sus características definitorias. Con base en esta interpretación se negaba la existencia de entidades materiales (muchas de ellas asociadas con construcciones mentales o intelectuales) que fuesen infinitas como totalidades acabadas. Surgen entonces, para recoger las interpretaciones que abogan en uno $\mathrm{u}$ otro sentido respecto al existir de lo infinito, las categorías de infinito en acto e infinito en potencia. La primera (que terminó siendo más formal, intelectual y elaborada) hace referencia a la existencia de seres o cosas infinitas, como totalidades abarcables mediante una "mirada con los ojos corporales" o a través de una "mirada envolvente con los ojos del pensamiento". La segunda no acepta la existencia de dichos entes como totalidades infinitas acabadas $\mathrm{o}$ encerradas sino como finitudes con posibilidades de expandirse o desarrollarse en un proceso sin fin.

En correspondencia con la concepción de lo infinito, como lo insondable e inabarcable, el pensamiento oficial acogió como válida, y ajustada a los hechos reales, la concepción del infinito en potencia. Esta escogencia tenía una significativa afectación ideológica pues no era conveniente, para el control de la comunidad por parte del Estado, permitir elucubraciones sobre una comprensión de las manifestaciones fenoménicas de la naturaleza por encima de los límites impuestos oficialmente al conocimiento, ni mucho menos aceptables algunas prácticas como la hechicería, la brujería y el ocultismo casi siempre al servicio de quienes están por fuera de los controles del estado. Sin embargo, por fuera de lo permitido y lo aceptado, se promovía una cosmovisión en contravía de la exagerada Racionalidad del pensamiento oficial. Dicha cosmovisión pregonaba un universo infinito perfectamente comprensible y abarcable por los seres humanos (Un infinito en acto) en el que podían evidenciarse todos los elementos constitutivos de la naturaleza, incluidos aquellos que, mantenidos como secretos por los grupos dominantes, se cubrían con un manto de misterio.

Ha sido, precisamente este manto de misterio, con el cual se ha cubierto la concepción del infinito, el que ha estimulado toda la fantasía que alimenta la creación literaria al respecto. Sin embargo, a diferencia de muchos otros Literatos, Borges, sin renunciar a su independencia intelectual y su extraordinaria capacidad para fantasear, recrea y da firmeza a sus ficciones con el aval de los rigurosos conceptos matemáticos. Claro está que comparando los trabajos de Cantor sobre el infinito con las disertaciones de Borges es difícil determinar si quien fantasea con relación al tema es Borges o Cantor.

La Paideia griega, referente básico del modelo educativo occidental, acoge el infinito en potencia, tal y como se evidencia en la axiomática euclidiana que nos presenta la línea recta (elemento primario en la composición y comprensión del espacio como entidad físico-geométrica) como un segmento limitado en sus extremos, con "posibilidad" de extenderse $a d$ infinitum prolongándose a partir de estos. 
Durante la Edad Media, bajo el severo control de la Iglesia Católica con la imposición de la escolástica, se mantuvo, más por inercia que por convicción, la concepción del infinito potencial. Hubo que esperar, mientras mejoraban las condiciones sociales y políticas y maduraban las condiciones intelectuales, en cuanto a la evolución de las bases teóricas, hasta el siglo XIX cuando se asume, por parte de los matemáticos alemanes, la tarea de "revisar" la fundamentación de la atemática formulada hasta ese momento.

Fue precisamente Cantor quien puso punto final a la histórica confrontación filosófica entre el infinito potencial y el infinito actual. No sólo fundamentó teóricamente la existencia del infinito en acto, sino que demostró que hay diferentes tipos de infinitos; con más rigor, diferentes órdenes de infinitud.

Antes de Cantor el término Infinito se usaba indistintamente para hacer referencia a todas las cosas, seres u objetos que fuesen infinitos en el sentido potencial; todo lo que fuese infinito era tratado con los mismos juicios de valoración. Este hallazgo de Cantor se dio a partir de una inquietud que venía de mucho tiempo atrás, incluso Galileo Galilei (1564-1642) estuvo a punto de resolverla. Se trataba de confrontar uno de los principios aristotélicos tomados como referente para todas las ciencias: "El Todo es mayor que cada una de sus partes".

Galileo llamaba la atención sobre la relación entre la serie o sucesión de los números naturales: $1,2,3,4,5,6,7,8 \ldots$ y la serie o sucesión de los cuadrados correspondientes a cada uno de los términos de dicha serie, a saber: 1, 4, $9,16,25,36,49,64 \ldots$ pues todo número natural tiene un cuadrado único (que es cuadrado perfecto) y, recíprocamente, todo número natural tiene una única raíz cuadrada que en el caso de los cuadrados perfectos corresponde al número natural del cual se obtiene dicho cuadrado (es importante aclarar que para entonces sólo se aceptaba y trabajaba con los números positivos). En tal sentido, los elementos de las dos series o sucesiones pueden colocarse en una correspondencia biunívoca, es decir, a cada elemento de la primera serie le corresponde un único elemento de la segunda serie y a cada elemento de la segunda le corresponde un único elemento de la primera. Este hecho nos permite deducir que, en cierto sentido, las dos series tienen igual cantidad de elementos. Sin embargo, y aquí viene lo verdaderamente interesante, la serie o sucesión de los cuadrados es parte de la serie o sucesión de los números naturales (obsérvese que en la primera serie hay "muchos", en realidad infinitos, números que no están en la segunda) y, en consecuencia, al tenor del principio aristotélico referido anteriormente, dicha serie o sucesión debiera ser menor, en cuanto a la cantidad de elementos, respecto a la sucesión de los naturales.

Situaciones como la anterior evidenciaban que "algo" no funcionaba bien en el diseño escolástico-aristotélico propuesto para abordar el estudio de las ciencias, en particular las Matemáticas; pero era mejor callar o trabajar en la clandestinidad manteniendo un bajo perfil. Ya Galileo había hecho suficiente ruido con sus estudios sobre el movimiento, hasta ridiculizar las bases ptoloméicas de la dinámica celeste aceptada por el clero, lo cual lo tuvo adportas de la hoguera inquisitorial después de haber sido sensibilizado en el "potro".

Casi tres siglos después de las disquisiciones de Galileo sobre la posibilidad de que dos conjuntos infinitos tengan la misma cantidad de elementos, la genialidad de Cantor dio un aparente salto al vacío y, de manera análoga a la cuantificación de la totalidad de los elementos de los conjuntos finitos (cardinalidad, se denomina hoy), para lo cual empleamos los números naturales (así, por ejemplo, el símbolo 5 , usualmente denominado número cinco, es el cardinal de todos los conjuntos, independiente de su naturaleza, que tienen cinco elementos), estableció la cardinalidad de los conjuntos infinitos (denominada cardinalidad transfinita) introduciendo nuevos números, que terminaron llamándose transfinitos, a los cuales asignó una 
simbología, los numerales, tomada del alfabeto hebreo. Para tan compleja y aventurada tarea, partió de los números naturales y se dio a la búsqueda de aquellos conjuntos infinitos cuyos elementos pudieran ponerse en correspondencia biunívoca con éstos. A tales conjuntos los consideró como los conjuntos Infinitos de primer orden por tener "igual cantidad" de elementos que el conjunto empleado como referente (los números naturales).

Fueron muchas las sorpresas que halló cantor en el desarrollo de esta búsqueda; algunas de ellas le "arrancaron" expresiones como: "Lo veo, pero no lo creo". Atrás quedó definitivamente, por lo menos en el mundo de lo infinito, el principio de que "el todo es mayor que cada una de sus Partes". En una especie de coadyuvancia al trabajo de Cantor, desde campos distintos a las matemáticas como el de las ciencias naturales, la filosofía y la lógica surgieron resultados que mostraban correspondencias biunívocas entre conjuntos infinitos aparentemente de tamaño o magnitud diferentes. El entusiasmo generado por los hallazgos de cantor produjo una especie de destape frente al infinito. Varios de estos resultados han sido recogidos como "las paradojas del infinito".

Seguramente convencido de su audacia, de la dimensión artística de su obra y, de tal vez, un poco de misticismo por develar los misterios del infinito, Cantor escogió para su presentación ante la comunidad matemática un simbolismo que estuviera a la altura de esta en cuanto a lo que dicha conceptualización representaba. En tal sentido, adoptó como numerales (o, símbolos) para representar sus números transfinitos la primera letra del alfabeto hebreo (para muchos un alfabeto sagrado, cabalístico), indexada de acuerdo al orden de magnitud del conjunto que cuantifique. Esta letra se conoce con el nombre de Aleph y se simboliza por: $\aleph_{0}$. Se dice que esta letra representa un hombre que apoya una mano sobre la superficie terrestre mientras con la otra señala la parte del universo donde se ubican los demás astros, indicando con este gesto que la tierra es un mapa que "recrea" dicho universo (no es gratuita, pues, la bola de cristal que, semejando el universo, es fuente de inagotable información para los adivinos y pregoneros del destino de las personas).

Como hay conjuntos infinitos de distinto orden (en relación con su magnitud) y el propósito de Cantor es clasificarlos y cuantificarlos a todos, respecto a la totalidad de sus elementos para establecer su cardinalidad, coloca subíndices a los Aleph para representar los números transfinitos que utiliza en dicho proceso. De acuerdo con esta notación o simbología, el primer cardinal transfinito es Aleph sub-cero $\left(\aleph_{0}\right)$, que corresponde al Cardinal de los números naturales. Es decir que el conjunto de los números naturales tiene Aleph sub-cero $\left(\aleph_{0}\right)$ elementos y, en consecuencia, todo conjunto que se pueda poner en correspondencia uno a uno con la totalidad de los naturales también tiene Aleph sub-cero $\left(\aleph_{0}\right)$ elementos. El conjunto del número reales, usualmente identificado con la línea recta, no se puede poner en correspondencia biunívoca con los números naturales; por lo tanto, estos dos conjuntos tienen diferente orden de magnitud. En cierto sentido, el conjunto de los reales es mayor que el de los naturales. El cardinal transfinito de los reales es Aleph sub-uno. En general, así como disponemos de los numerales $1,2,3,4,5,6,7,8,9,10 \ldots$ para cuantificar los conjuntos finitos que tienen uno, dos, tres, cuatro, cinco, seis... elementos, Cantor introdujo los numerales $\aleph_{0}, \aleph_{1}, \aleph_{2}, \aleph_{3}, \ldots$ para cuantificar los conjuntos infinitos que tienen Aleph sub-cero, Aleph sub-uno, Aleph sub-dos, Aleph sub-tres... elementos.

Una vez proveído de estos recursos, el proyecto cantoriano avanzó hacia la construcción de una aritmética transfinita que debía recoger y superar situaciones relativamente elementales en el campo de lo finito, las cuales se hacen terriblemente complejas e incomprensibles en el mundo de lo Infinito. No fue nada fácil para Cantor entregarle a la posteridad tan maravillosos y valiosos elementos de una obra monumental que en su momento no halló la suficiente comprensión de los académicos rectores 
del ordenamiento curricular autorizado en los programas oficiales de estudio y enseñanza en colegios, institutos y universidades. Los continuos ataques a su objeto de investigación, la deslegitimación de sus resultados y el bloqueo sistemático de su difusión desde la lógica argumentativa de la fuerza que confiere el ejercicio del poder, hicieron mella en su salud mental obligándolo a permanecer largas temporadas en clínicas de reposo y hospitales psiquiátricos alejado de la actividad investigativa que llenaba su existencia.

Leopold Kronecker, quien había sido profesor de Cantor en la Universidad de Berlín, fue el mayor contradictor de Cantor en relación con $\mathrm{su}$ trabajo sobre el infinito. Desconociendo el trabajo de Cantor quiso despachar de la Matemática los cardinales transfinitos (los Aleph) con una afirmación que terminó haciéndose famosa fuera del contexto original: "Dios hizo los números naturales". Es decir, que con ellos bastaba en materia de números siendo innecesario, pues, crear o construir nuevos números, ya que la divina providencia le había dado al hombre, para que hiciera lo demás (que era todo), los números naturales. De esta manera, desautorizó a Cantor con un imperativo categórico divino.

Tal vez, teniendo en cuenta que Cantor empleó la letra Aleph del alfabeto hebreo para representar los numerales correspondientes a los cardinales de los conjuntos Infinitos, Borges retoma dicha letra para nombrar aquellos objetos milenarios que recreó en sus ficciones, cuentos y relatos en los cuáles, se decía y se creía, estaba absolutamente todo lo que fuera o pudiera ser objeto de conocimiento, todo lo que existirá o pudiera existir.

Así, la bola de cristal del adivino, la vara mágica del hada madrina, la capa del mago, los espejos de los cuentos de hadas, el genio de la lámpara de aladino, las vísceras de las aves que utiliza el agorero, la bolsa de fortunatos, todos ellos pueden ser Alephs.
De hecho, en uno de sus más afamados y estudiados relatos o ficciones: "El Aleph", Borges reconoce que era un falso Aleph el objeto oculto en el sótano de la casa habitada por Carlos Argentino en la calle Garay a través del cual

[... vi la tierra, y en la tierra otra vez el Aleph y en el Aleph la tierra, vi mi cara y mis vísceras, vi tu cara y sentí vértigo y lloré, porque mis ojos habían visto ese objeto secreto y conjetural, cuyo nombre usurpan los hombres, pero que ningún hombre ha mirado: El inconcebible universo. p. 62, 1983

Él considera que hay (o que hubo) otros Aleph: el espejo de Alejandro Magno en cuyo cristal se reflejaba el universo entero; la séptuple copa de Kai Josrú; el espejo que Tárik Benzeyad encontró en una torre; el espejo que Luciano de Samasota pudo examinar en la luna; la lanza especular que el primer libro del Satyricón de Capella atribuye a Júpiter; el espejo universal de Merlín, "redondo y hueco y semejante a un mundo de vidrio". Sin embargo, con base en la extensa y curiosa bibliografía que soporta sus ficciones, manifiesta que todos los anteriores objetos (aparte del defecto de no existir) son meros instrumentos de óptica.

Resulta estimulante que una teoría, que al ser vertida en el molde formal de la matemática se torna rígida, esquemática, exigente $\mathrm{y}$ prácticamente inabordable, pueda ser recreada, sin desvirtuar su esencia, de forma tan amena, tan atractiva y provocadora como lo ha sido el concepto de infinito actual matemático a través del ejercicio literario de Borges.

Mucho habrá que reflexionar sobre la relación que existe entre Matemática y Literatura en beneficio de la creación científica y cultural. Así como conocemos a través de la lógica la significativa relación entre el pensamiento matemático el lenguaje y la escritura, podremos conocer, mediante la libre creatividad, la relación que existe entre estos dos campos del conocimiento. 


\section{Referencias}

Borges, J. L. Historia Universal de la Infamia. (1983). Bogotá, Colombia: Editorial la Oveja Negra Ltda.

Borges, J. L. El Aleph. (1983). Bogotá, Colombia: Editorial La Oveja Negra Ltda.

Borges, J. L. El Hacedor. (1983). Bogotá, Colombia: Editorial Oveja Negra Ltda.

Borges, J.L. Prosa. (1985). Barcelona, España: Industria Gráfica San Vincens Dels Hors.

Bourbaki, N. (1972). Elementos de Historia de la Matemática. Madrid, España: Editorial Alianza Editorial.

Castro Chadid, I. \&, Pérez Alcázar, J. H. Un paseo Finito por el Infinito, el infinito en
Matemáticas. Bogotá, Colombia: Pontificia Universidad Javeriana.

Gutiérrez Girardot, R. (1998). Jorge Luis Borges, el Gusto de ser Modesto. Bogotá, Colombia: Panamericana Editorial.

Pinilla, A. (2004). Jorge Luis Borges La Literatura Como Tierra Propia. Bogotá, Colombia: Panamericana Editorial.

Real Academia Española. (2017). Borges Esencial. Lisboa, Portugal: Talleres Gráficos de Printer Rio de Mouro.

Borges, J. L. Borges Obras Completas. (1974). Buenos Aires, Emecé Editores. Recuperado de: https://literaturaargentina1unrn.files.wo rdpress.com/2012/04/borges-jorge-luis-obras completas.pdf, (noviembre 17 de 2017). 


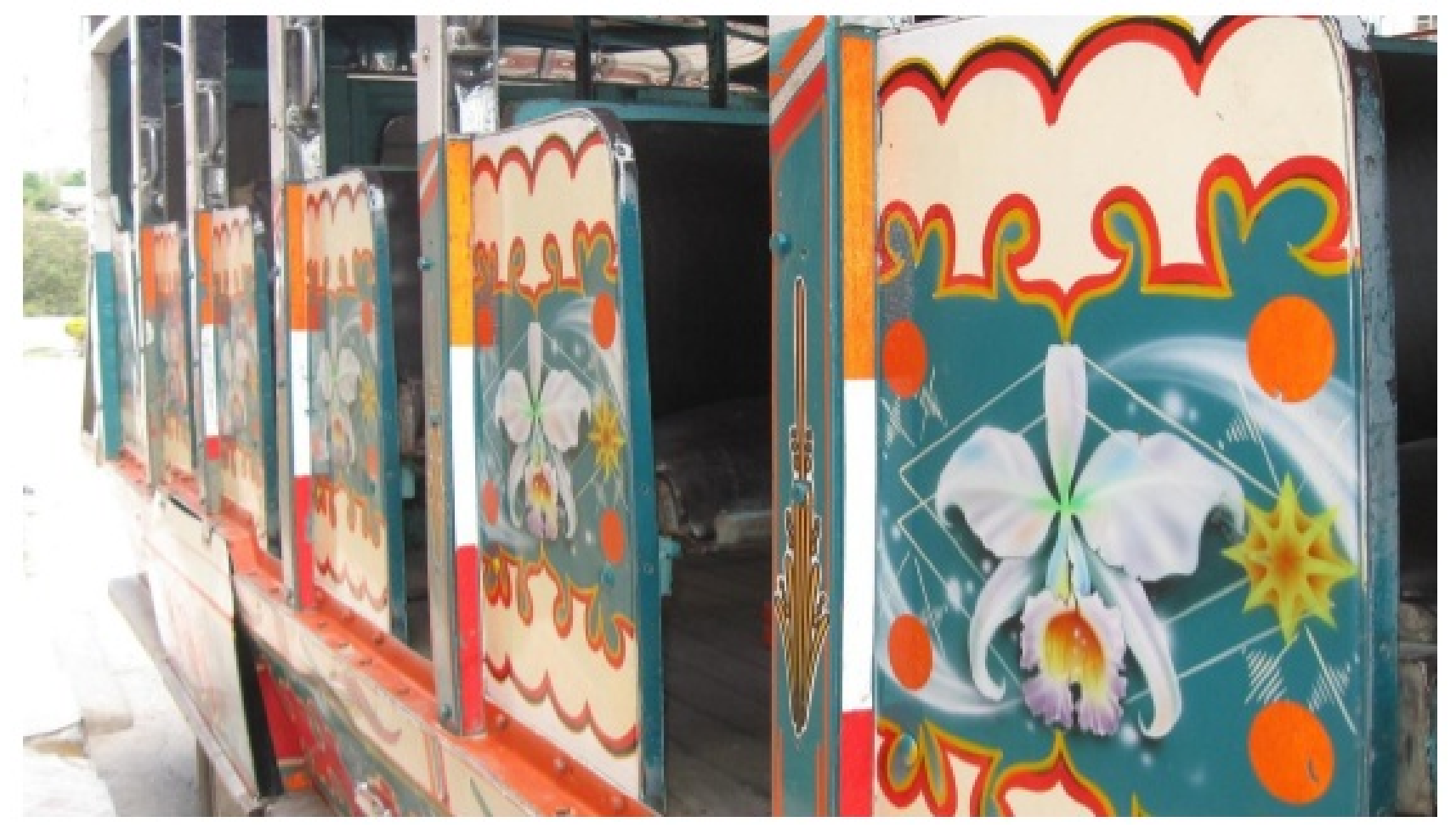

Número de la obra: 77

Título: Chiva "El Lucerito"

Autora: Margarita Chávarro Castro

Lugar: Municipio La Plata, Huila

Fecha de captura: Abril de 2013

Técnica: Fotografía digital

Fuente: Colección Margarita Chávarro Castro 